\title{
Research of multi-rotor UAV atmospheric environment monitoring system based on 4G network
}

\author{
LIU Xiaojun ${ }^{1}$, WEN Hongyuan ${ }^{1}$, LI Ao $^{2}$, XU Di ${ }^{3}$, HOU Zhengxuan ${ }^{1}$ \\ ${ }^{1}$ Taizhou Institute of Sci.\& TECH., NUST., Taizhou, Jiangsu 225300, China; \\ ${ }^{2}$ Taizhou Blue Sea Environmental Protection Technology Co., Ltd, Taizhou, Jiangsu 225300, China; \\ ${ }^{3}$ Yangzhou University, Yangzhou, Jiangsu 225000, China
}

\begin{abstract}
Aiming at the shortcomings of the traditional fixed atmospheric environment monitoring system, such as low flexibility, limited monitoring space, and the limitation of the distance of the unmanned aerial vehicle (UAV) atmospheric environment monitoring based on the data transmission module and the limited amount of data transmitted online, the multi-rotor UAV atmospheric environment monitoring system transmitted by $4 \mathrm{G}$ network is designed, transmits the atmospheric data collected by the airborne atmospheric detection sensors to the network cloud server through the $4 \mathrm{G}$ network, and realizes real-time monitoring and analysis of atmospheric data on the data monitoring platform, combined with the transparent cloud and cloud configuration technology. Firstly, the shortcomings of traditional ground monitoring stations and drone monitoring methods were analyzed, and the overall design of the system is given; then, the software and hardware design of the system were expounded in detail; finally, experiments were carried out in in the university town of Hailing area of Taizhou city. The research results can effectively expand the working space and methods of traditional atmospheric environment monitoring and provide new ideas and methods for the research of environmental monitoring UAV.
\end{abstract}

\section{Introduction}

Air pollution has long been a global concern. The harmful gases in the ambient air affect people's health and the safety of some production work. Therefore, it is of great importance and significance to master the composition and concentration of harmful gases in the atmosphere in real time. At present, the main means of atmospheric monitoring are fixed monitoring on the ground. As an emerging industry ${ }^{[1]}$, UAV technology has been widely used in agriculture ${ }^{[2]}$, forestry ${ }^{[3-4]}$, terrain mapping ${ }^{[5-6]}$, electricity ${ }^{[7]}$, disaster monitoring and environmental monitoring ${ }^{[8-10]}$.

With the rapid development of micro-electromechanical systems (MEMS), micro-inertial measurement unit (MIMU) and other technologies, the atmospheric environment monitoring technology of multi-rotor UAV has gradually been favoured by more scholars ${ }^{[11-12]}$. Chen chen used UAV technology to construct the diffusion model of atmospheric pollution sources, so as to realize the tracking and positioning of atmospheric pollution sources ${ }^{[13]}$. Chen yizhou designed a mobile monitoring system for urban air quality based on UAV and low-cost sensors, and used GPRS to transmit data to obtain spatial and temporal distribution characteristics of air pollutants in specific areas ${ }^{[14]}$. Yang guolin integrated the UAV technology with the Beidou Navigation System, and completed the detection and recording of the air micro-particulate matter, temperature and humidity in specific locations in the space ${ }^{[15]}$. However, few literatures mention the real-time monitoring of atmospheric gases by combining $4 \mathrm{G}$ network with UAV technology. Therefore, this paper proposes a multi-rotor UAV air environment monitoring system based on $4 \mathrm{G}$ network, which transmits the atmospheric data collected by the airborne atmospheric detection sensors to the network cloud server through the $4 \mathrm{G}$ network, realizes real-time monitoring and analysis of atmospheric data, combined with the transparent cloud technology, solves the problems of the limitation of the distance of air quality monitoring of traditional UAVs and the limitation of the amount of data transmitted online, and provides new ideas and methods for the research of UAV environmental monitoring.

\section{Overall system design}

The air environment monitoring system of UAV based on $4 \mathrm{G}$ network designed in this paper mainly includes two parts: multi-rotor UAV subsystem and air monitoring subsystem. The multi-rotor UAV subsystem, as an air flight platform, mainly includes flight control unit, motor and drive, power supply/rack unit, flight management unit and other modules. The atmospheric monitoring subsystem plays a role in the detection,

\footnotetext{
*Corresponding author: 93429706@qq.com
} 
transmission and display of atmospheric data, including atmospheric detection sensor, $4 \mathrm{G}$ network transmission module, network cloud server, transparent cloud and data monitoring platform. The system structure diagram is shown in figure 1 .

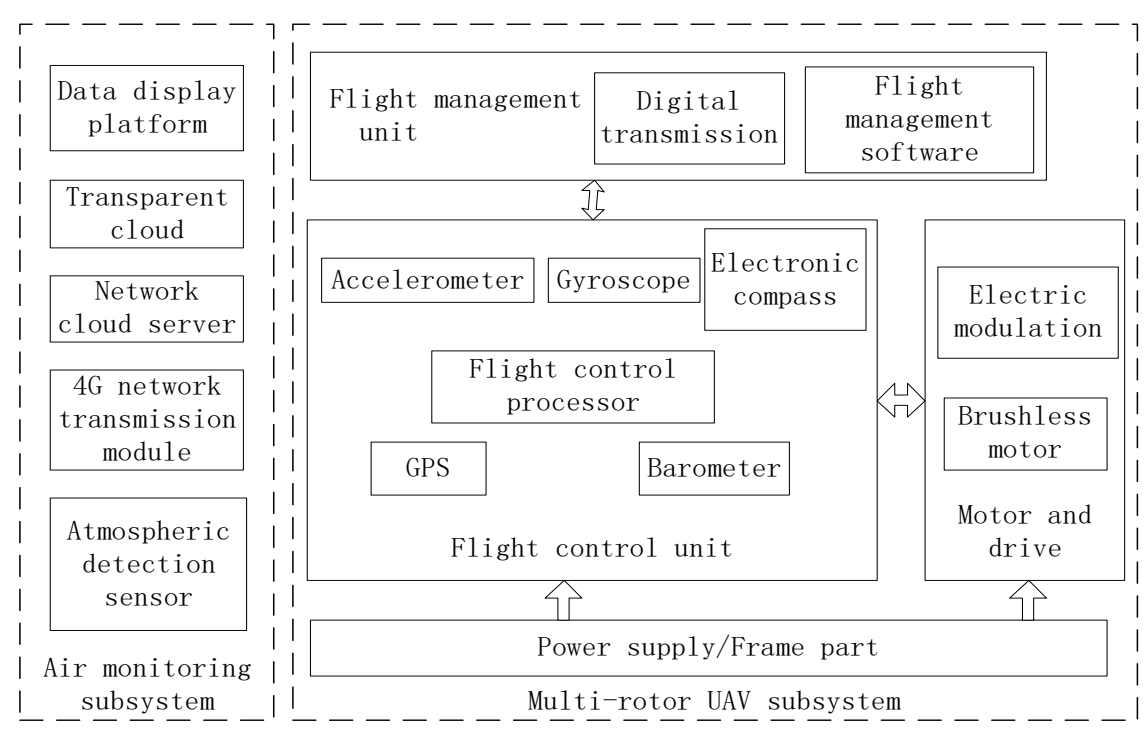

Fig.1. Structure diagram of UAV atmospheric environment monitoring system based on 4G network

\section{System hardware design}

\subsection{Multi-rotor UAV subsystem}

\subsubsection{Flight control unit}

Flight control unit is the core part of multi-rotor UAV, the need to manage all parts of the UAV system as a whole, through a series of complex operations: gyroscope, digital compass sensor data, carries on the attitude algorithm, based on digital transmission module of instruction, combined with the attitude control algorithm, control of each motor output signal, no extension of the flight attitude and position control. There are many kinds of flight control used in the market, most of which are expensive, and the software and hardware are not open source. In this paper, STM32F407 processor chip is adopted, equipped with peripheral circuits such as gyroscope and digital compass, and flight control unit is independently developed. After flight test, the performance is good and stable, the flight control unit $\mathrm{PCB}$ is shown in figure 2 .

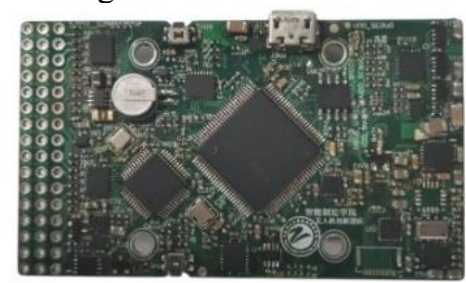

Fig.2. The flight control unit $\mathrm{PCB}$

\subsubsection{Rack}

The rack is the mounting support part of the flight control unit, battery, electrical modulation, air

acquisition, transmission module, picture transmission and other modules, mainly including the body of the rack, the arm and other parts. Considering the weight of the airborne load, a six-rotor frame with wheelbase 830 is designed in this paper, which can install 6 brushless dc motors. The frame design drawing is shown in figure 3. The machine arm is mainly used as the installation support part of the blade, motor and other components. It adopts a foldable design to facilitate operation. The design drawing is shown in figure 4 . The main body of the frame is designed in hexagonal shape, which is divided into two parts: upper plate and bottom plate, which are cut by carbon fiber plate. The upper and lower plates are fixed with internal Angle screws. Flight control unit, receiver, digital transmission and electrical adjustment are installed in the middle. The upper and lower board 3D printing and physical drawing are shown in figure 5 , figure 6 , figure 7 and figure 8 .

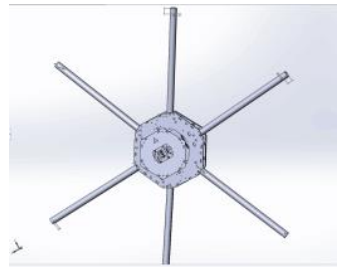

Fig.3. The design drawing

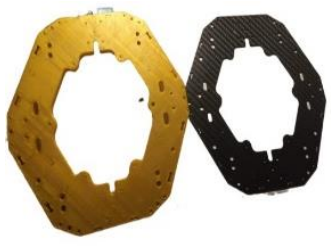

Fig.5. The upper board

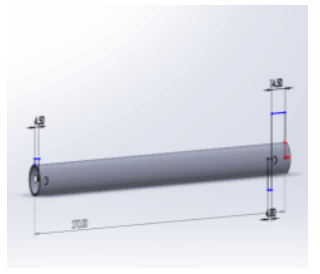

Fig.4. The design drawing

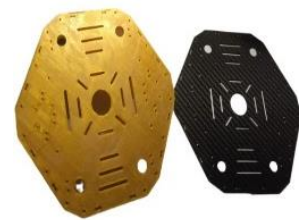

Fig.6. The lower board 


\subsubsection{Motor and drive unit}

The motor and drive unit include brushless de motor and electronic modulation. The electronic modulation outputs PWM control signal according to the command signal of the remote control and the flight control unit, so as to realize the speed regulation of the motor and the attitude control of the UAV. This paper adopts haoying 40A electrical modulation with high cost performance and stable operation, and six hurricane kv420 brushless motors, as shown in figure 7 and figure 8 .

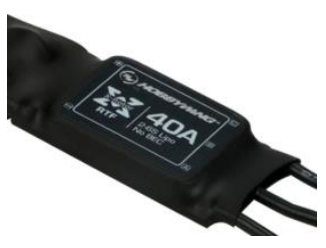

Fig.7. Electronic modulation

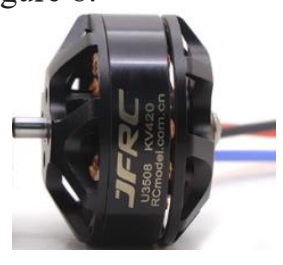

Fig.8. Brushless motor

\subsection{Air monitoring subsystem}

Atmospheric monitoring subsystem mainly includes atmospheric detection sensor, 4G network transmission module, network cloud server, transparent cloud and data monitoring platform. The $4 \mathrm{G}$ network transmission module is used to realize the communication between the atmospheric detection sensor and the network cloud server. The function of supporting access to the transparent cloud is the core of the whole system. The transparent cloud enables transparent transmission of data between $4 \mathrm{G}$ modules and network servers, without changing any structure or data, and the whole process is transparent to the outside world. Through the virtual serial port, pass-through cloud platform establishes communication with the network cloud server and data monitoring interface, solves the data communication between gas detection and the upper PC, and realizes the function of remote monitoring. The work flow chart is shown in figure 9.

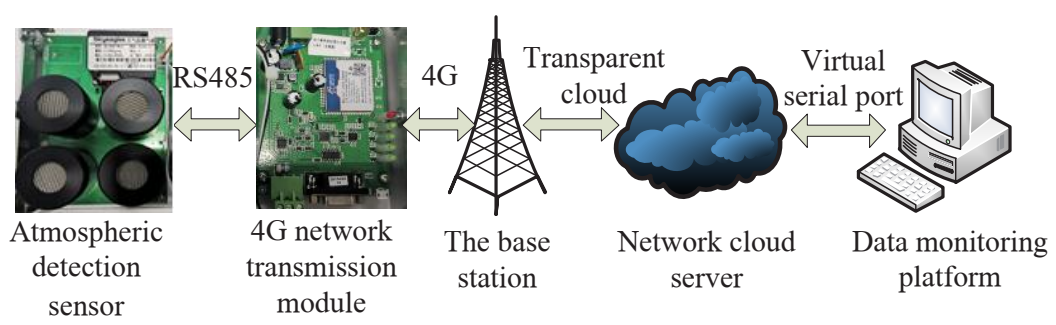

Fig.9. Working flow chart of air monitoring subsystem

Among them, the atmospheric detection sensor and 4G network transmission module are the main parts of the hardware. The integrated closed structure design is adopted, and the top layer of the equipment is perforated to facilitate gas circulation, so that the measurement results are more accurate, as shown in figure 10 and figure 11. The atmospheric detection sensor module mainly includes NO2, SO2, O3, CO, PM2.5 and PM10 gas and particle concentration sensors, which adopt a detachable structure so that other gas concentrations can be detected at a later stage. The gas detection resolution reaches PPB level, which ensures the accuracy of data detection. The $4 \mathrm{G}$ network transmission module uses a high-speed, low-latency full Netcom $4 \mathrm{G}$ module, which supports network transparent transmission, protocol (UDC) transparent transmission and other working modes, and the data transmission is stable and efficient.

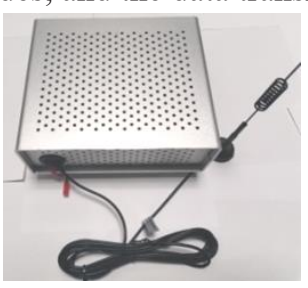

Fig.10. Appearance

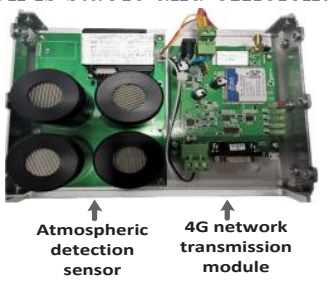

Fig.11. Internal structure

\section{System software design}

\subsection{Flight control software}

The stability of UAV control system requires not only the hardware part of flight control unit but also the software control part. In this paper, double closed-loop cascade PID control algorithm is adopted, combined with complementary filtering algorithm and four-element attitude solution, so that the UAV control system has good stability and robustness. The system control software program is shown in figure 12 .

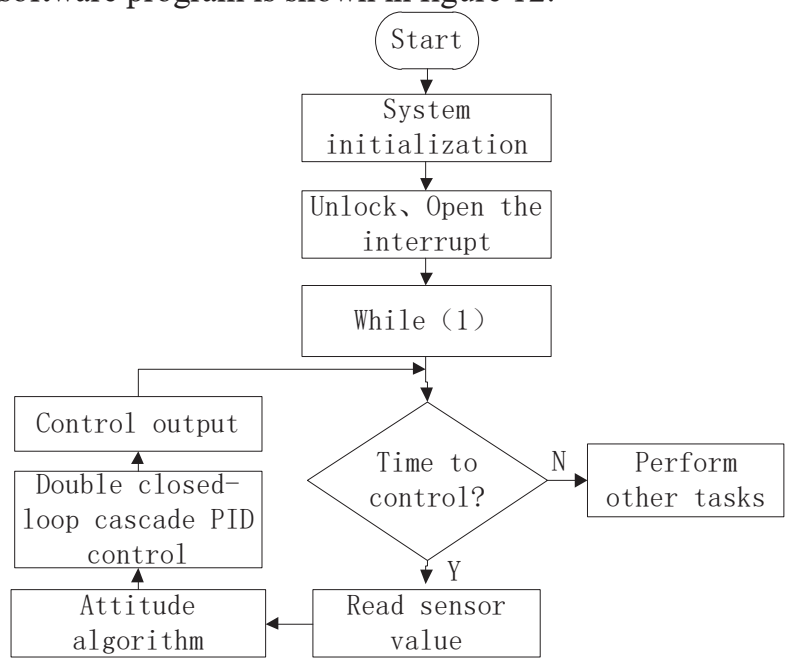

Fig.12. Flight control system software design flowchart

The attitude adjustment of the six-rotor UAV is realized by adjusting the speed of the six motors. Different speed combinations can realize the different flight attitude of the UAV, such as hover, rise and 
advance. By designing the PID control law in the three attitude control channels, the required control function can be realized. The traditional PID algorithm can easily cause the system to vibrate and cause the aircraft to deviate from the steady state. This paper adopts a double closed-loop cascade PID control algorithm, loop controller of attitude Angle adjustment precision, rapid changes in the velocity of the inner loop controller diagonal trend of adjustment, improve the system dynamic control under the disturbance, guarantee the stability of the system control performance and flight, flight control block diagram as shown in figure 13 .

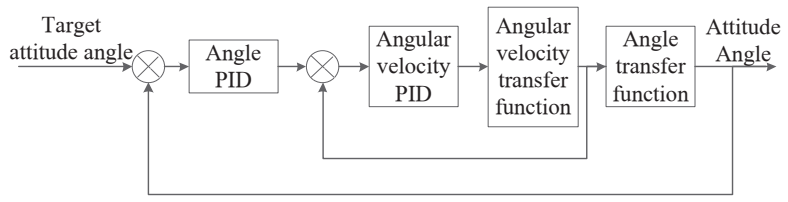

Fig.13. Block diagram of double closed-loop control structure

\subsection{Flight management software}

Flight management software, also known as ground station, the function is for UAV flight control, takes through the UAV route planning as well as to the UAV flight attitude data of observation, which can help to complete safe flight. Based on the open-source software mission planner, this paper uses $\mathrm{C \#}$ language for secondary development, which has multi-function, covering debugging, flight, simulation and other functions in an all-round way.

\subsection{Data monitoring platform}

The development of the data monitoring platform is based on the cloud configuration technology, which can accomplish real-time display of atmospheric detection sensor data, alarm push, group management, historical data view and other functions in a one-stop manner. The large monitoring screen can show the distribution of running equipment on the map, as well as equipment overview, alarm information, etc.

\section{Test and performance analysis}

The test process is divided into three stages: UAV subsystem test, atmospheric detection subsystem test and system overall joint test.

UAV subsystem test: Firstly, download the flight control program to the flight control unit, connect the ground station with USB cable, observe the attitude data of the UAV through the ground station software in real time, and test the software and hardware performance of the flight control unit. Then the electric harmonic motor is tested, including electric adjustment pair, motor positive and negative rotation, etc. Finally, the UAV subsystem is tested, and the PID control parameters are adjusted through outdoor flight to ensure the flight stability of the UAV platform.
Atmospheric detection subsystem test: Firstly, the serial port of the atmospheric detection sensor module is directly connected to the serial port of the computer, so that the monitoring data of each air sensor can be directly sent to the computer for viewing, so as to conveniently observe the output parameters of the sensor. Then connect the $4 \mathrm{G}$ network transmission module to the atmospheric detection sensor module with RS485 bus, set up the pass-through cloud platform, add equipment, and view the data of the atmospheric sensor in real time through the data monitoring platform.

System overall joint test: After the completion of the testing of the UAV subsystem and the air detection subsystem, the air sensor detection equipment is installed on the UAV (horizontal center of gravity) for joint testing of the whole system, as shown in figure 14 and figure 15 .

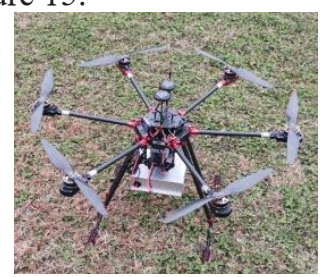

Fig.14. Physical picture

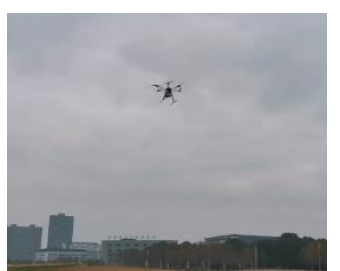

Fig.15. Outdoor test chart
The test was carried out near the university town of hailing district, Taizhou city. The atmospheric detection sensor collected and sent data every $4 \mathrm{~s}$, and checked the data waveform and historical data in real time on the atmospheric monitoring platform, as shown in figure 16 and figure 17.

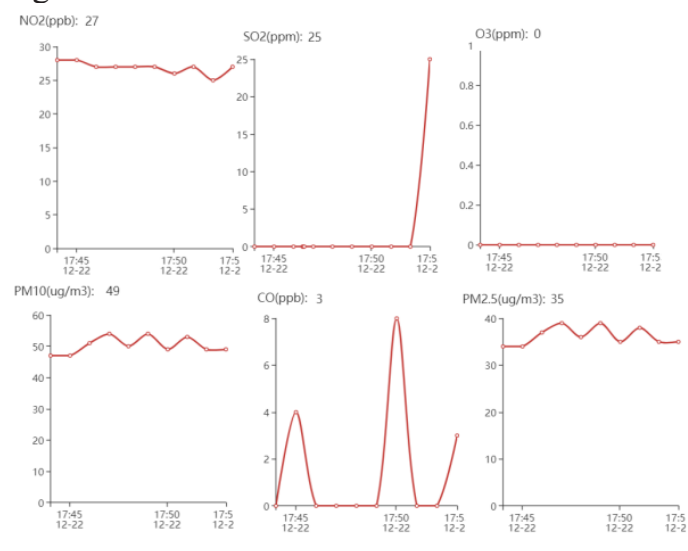

Fig.16. Real-time data display

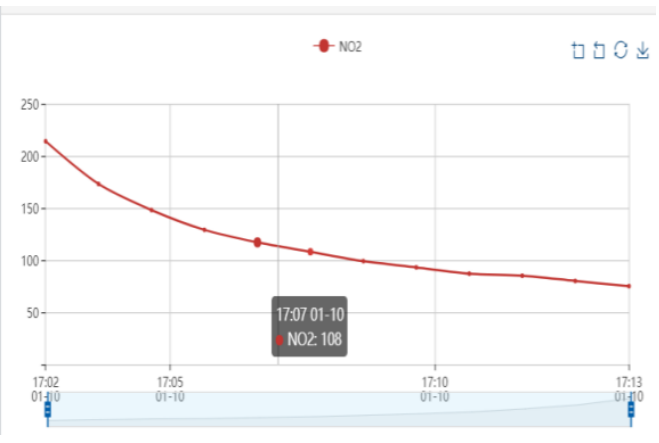

Fig.17. Historical data 
In order to test whether the performance of the system can meet the expected indicators, the system compares the gas concentration, particulate matter concentration, position information and other data collected in multiple sets of different environments with special equipment. The test results show that the errors of the gas concentration, particle concentration and location parameters obtained by the system are all within the acceptable range, which can be used as an effective means for atmospheric environmental protection research and other works.

\section{Conclusion}

This paper designs and develops a multi-rotor UAV atmospheric environment monitoring system based on $4 \mathrm{G}$ network. Utilizing the high mobility of the drone and the high-speed coverage of the $4 \mathrm{G}$ network to achieve dynamic detection of the six parameters of the national standard, real-time monitoring and analysis of atmospheric data through the transparent cloud and cloud configuration data monitoring platform. This system makes up for the shortcomings of the traditional fixed air quality monitoring system, such as low flexibility and limited monitoring space. It provides multiple monitoring methods such as real-time monitoring and detection of dangerous areas, and solves the air quality monitoring distance of drones based on digital transmission chains. The problem of limited and online data transmission has increased the diversity and reliability of urban air quality monitoring, and provided new ideas and methods for research on environmental monitoring of UAV.

\section{Foundation support:}

Jiangsu University Blue Project (No.201812); Taizhou city science and technology support program (social development) project (No. TS201819)

\section{Reference}

1. Li Deren, Li Ming. Research progress and application prospect of UAV remote sensing system $[\mathrm{J}]$. Journal of Wuhan University (Information Science Edition), 2014, 39(05): 505-513+540.

2. Deng Wenwen. Application research of farmland detection system based on UAV spectral analysis[J]. Research of Agricultural Mechanization, 2020, 42(07): 119-124.

3. Huang Bei, Zhu Liyan, Liu Wenguo. Inspection and application analysis of forest land management based on UAV imagery[J]. Forestry Resource Management, 2017(06): 153-158.
4. Xie Bing, Yang wunian, Wang Fang. A new method for estimating vegetation coverage based on UAV visible light spectrum[J/OL]. Science of Surveying and Mapping, 2020(09): 1-10.

5. Zhang Chunbin, Yang Shengtian, Zhao Changsen, Lou Hezhen, Zhang Yichi, Bai Juan, Wang Zhiwei, Guan Yabing, Zhang Yuan. Verification of terrain data accuracy for small consumer UAV[J]. Journal of Remote Sensing, 2018, 22(01): 185-195.

6. Wang Zhongxiang, Wu Hao, Zhu Jie, Zhang Ronghui, he Zihao, Chen Lihong. Study on the emergency mapping system of industrial UAV[J]. Science of Surveying and Mapping, 2019, 44(07): 177-182.

7. Zhao Ye long, Ji Chang Dong, Du Quan Ye. Digital orthophoto map making based on UAV electric power line inspection[J]. Science of Surveying and Mapping, 2018, 43(09): 146-152

8. Huang Haining, Huang Jian, Zhou Chunhong, Pan Yongjie. Application of drone image in survey of dangerous rock mass on high and steep slopes[J]. Hydrogeology \& Engineering Geology, 2019, 46(06): 149-155.

9. Hou Tianhao, Xing Hongyan, Liu Yang. The status and prospect of multi-rotor UAV in meteorological detection[J]. Journal of Electronic Measurement and Instrumentation, 2019, 33(10): 7-15.

10. Yang Wei, Shan Chunyan, Liu Junbo. Underground unmanned aerial vehicles cluster system for monitoring and its key technologies[J]. Industry and Mine Automation, 2019, 45(04): 6-12.

11. Peng Yan, Ye Jinwei, Zhang Liyong, Nie Chenhui, Ni Huizhu, Zhang Aomin. Study on three dimensional air quality monitoring by unmanned aerial vehicle[J]. Science of Surveying and Mapping, 2017, 42(11): 154-157+170.

12. Liu X, Li B, Jiang A, et al. A bicycle-borne sensor for monitoring air pollution near roadways[C]// 2015 IEEE International Conference on Consumer Electronics-Taiwan (ICCE-TW). IEEE, 2015.

13. Chen Chen, Yang Jing, Wu Liang. Simulation of atmospheric pollution source tracking and positioning under UAV remote sensing monitoring[J]. Computer Simulation, 2019, 36 (06): 32-35.

14. Chen Yizhou, Lin Qiguang, Xia Jing, Xiang Chaosheng, Hang Fubing, Liu Xiaofeng. Drone-borne mobile monitoring system for urban air quality[J]. Journal of Electronic Measurement and Instrument, 2019, 33(10): 1-6.

15. Yang Guolin, Zhang Jianhuan, Han Feng, Liu Tao, Yue Zhilong, He Jiaqi. Integrated design of air quality monitoring system based on BDS[J]. Experimental Technology and Management, 2018, 35(06): $73-76+82$. 